\title{
Getting more out of your images: Augmenting photos for recollection and reminiscence
}

\author{
Florian Güldenpfennig \\ Vienna University of Technology \\ Favoritenstr. 9-11, A1040 Vienna \\ florian.gueldenpfennig@tuwien.ac.at
}

\author{
Geraldine Fitzpatrick \\ Vienna University of Technology \\ Favoritenstr. 9-11, A1040 Vienna \\ geraldine.fitzpatrick@tuwien.ac.at
}

\begin{abstract}
In recent years $\mathrm{HCl}$-related research has shown an increasing interest in systems designed for supporting human memory. However, many of these systems focus more on the technical challenges, especially around data collection and retrieval. In this paper we introduce three ideas for augmenting digital images to support recollection, reminiscing and reflection. Three playful applications are proposed that are motivated by related theories in psychology. They employ experimental concepts by modifying images in different ways before displaying them and hence adding additional value. Our intention is to activate the observer's memory, to focus on the detail of things and to take a look "behind the camera". We propose that such approaches can encourage more mindful engagement with the huge amount of digital images available and promote positive effects linked to reminiscing.
\end{abstract}

Digital photography, use of images, reminiscence, recollection, reflection, camera-phone, play

\section{INTRODUCTION}

The ability to capture digital images is increasingly becoming a part of our everyday life, enabled by the ready affordability of digital cameras. The inclusion of a built-in camera with new cell phones increases this ubiquity even more so. While this can lead to an overwhelming number of images to manage and make sense of, it also creates opportunities to explore more provocative and meaningful engagement with images.

In this paper, we present prototypes of three playful applications - EyeforDetail, ForgetMeNot and BehindTheCamera - for working with images and report results of pilot studies. Here, we are primarily concerned with supporting notions of recollection, reminiscence and reflection, topics that have recently started to receive some attention within $\mathrm{HCl}$ and for which images hold particular relevance. The common concept across the applications is to use image manipulation, motivated by related theories in psychology, to open up new perspectives and thus new opportunities for recollection, reminiscence and reflection for the observers. We go on to review $\mathrm{HCl}$ research related to images and to define what we mean by recollection, reminiscence and reflection before describing the applications.

\section{RELATED WORK}

$\mathrm{HCl}$ research has addressed numerous questions regarding photography and digital images. Works span topics such as the organization of image collections (Kirk et. al. 2006), the sharing of images (Frohlich et. al. 2002), augmenting images with additional information e.g. associated audio files (Frohlich et. al. 2000) or GPS coordinates, and many more. Various efforts have also been taken to facilitate image retrieval and bespoke devices have been prototyped for displaying digital images, e.g. (Banks and Sellen 2009). Our approach is similar to the augmentation work but explores different image manipulation approaches for recollection, reminiscence and reflection.

According to Sellen and Whittaker (2010, p.73), recollection denotes detailed thinking back to past personal experiences and comprises the remembering of facts such as "what clothes did I wear on a certain occasion." Reminiscing incorporates emotional aspects into recollection. It is more of a dwelling in the past, often times experienced as pleasurable. In contrast, reflection is about revisiting past events and viewing them from another perspective, often to learn from experience. This might for example lead to the discovery of underlying behaviour patterns. 


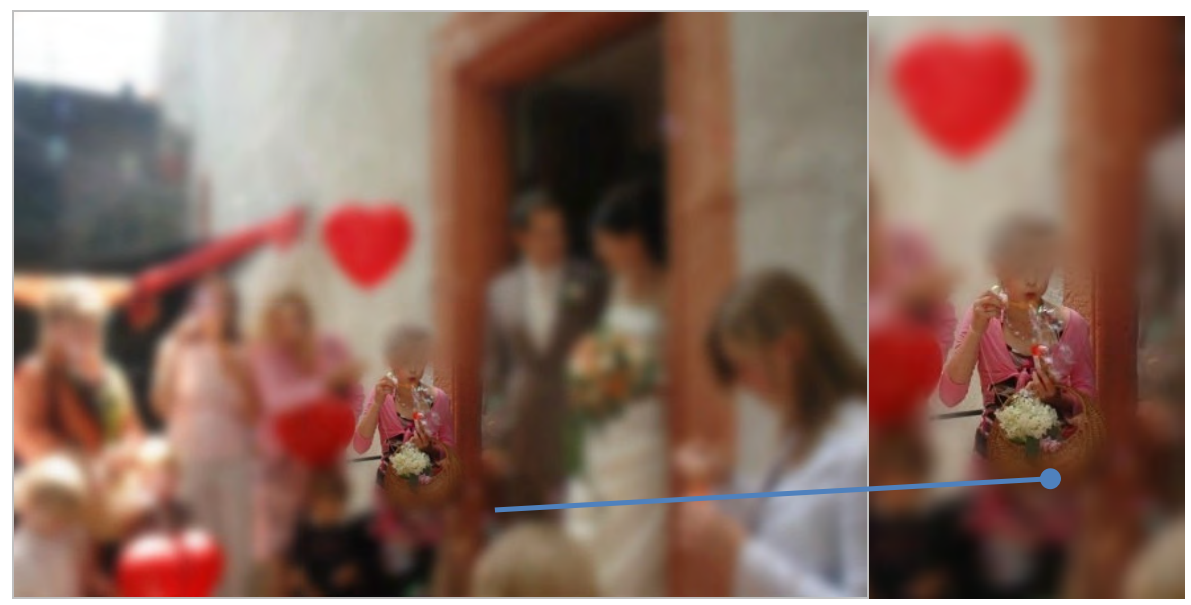

Figure 1: EyeOfDetail image viewer. Colourful marriage-scene (left) and magnified detail (right).

Recollection or revisiting of events constitutes the first step in memory consolidation and thus necessarily precedes reminiscence as well as reflection (Fleck and Fitzpatrick, 2009, 2010).

A number of systems have been developed to support recollection, reminiscence and reflection. Digital images play an important role in many of them, e.g. (Fleck and Fitzpatrick, 2009, Peesapati et. al. 2010, Sellen et. al. 2007, Uriui et. al. 2009). Our concepts are also based on the potential of images to evoke memories. Sellen and Whittaker (2010) point out that many lifelogging systems, which capture images and other personal data, primarily focus on technical collection and retrieval challenges and neglect how the user engages with the images and data. Here we focus on this engagement and aim to provoke recollection, reminiscence and reflection by augmenting digital images before displaying them to help the observer gain new insights.

We present three playful software applications, namely EyeforDetail, ForgetMeNot and BehindTheCamera. The first two applications take existing photos and apply modifications to them. BehindTheCamera attaches extra information to new images. The design of these applications was framed by two main concerns: that they be at low technical cost; and that the design concepts be motivated by relevant theories from psychology.

We propose that such approaches are worth exploring because of (1) the huge number of digital images available and (2) the positive effects that are linked to reminiscence. Reminiscing can be pleasurable, but it is also important for self/group identity (Webster and McCall 1999) and learning from experience (Fleck and Fitzpatrick 2009). It is also assumed to have an adaptive function (Havinghorst and Glasser 1972).
In the following section the applications are introduced. We then report on pilot studies of each of these in use and finally point to opportunities for further research.

\section{THREE EXPERIMENTAL APPLICATIONS}

\section{EyeOfDetail (image viewer)}

EyeOfDetail is the attempt to create software that in a way maps and supports the concept of mindfulness. In philosophy and psychology, mindfulness is most commonly defined as a state of being attentive and aware of the present moment and of what is taking place around us (Brown and Ryan 2003, p.822). Higher levels of this distinct form of awareness and attention can have strong positive effects on wellbeing (Langer 1989) and are associated with less emotional and cognitive disturbance (Brown and Ryan 2003).

The overall intention then of EyeOfDetail is to slow the observer down for a while, to focus attention and to encourage them to spend time, and deal indepth, with the moment that is captured in the image. The application loads an image from data memory, blurs the pixels by Gaussian filtering and displays the modified content on a common LCD screen. While at first sight there is a loss of image quality and data, we hypothesise that the observer will actually be able to get more out of the image. To accomplish this, one small spot of the image is left un-blurred. This clearly visible spotting window is designed to focus the observer's attention to one area at a time.

The setup of EyeOfDetail allows the user to coarsely navigate the position of the spot by wobbling an accelerometer device (see Figure 2). Thus, the observer is capable of controlling what part of the image can be clearly examined. In doing 


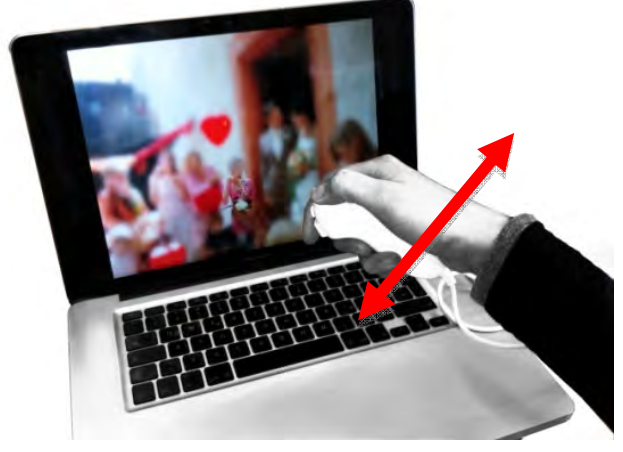

Figure 2: Navigating the clear spot of EyeOfDetail.

so details can be discovered that would have been dwarfed by more prominent content of the original image. We choose to give the user control of the spot by an accelerometer in order to provide a different experience from the common screenmouse interaction.

Figure 1 displays a snapshot of the EyeOfDetail image viewer. The image shows a marriage scene of rich colour and detail. Due to the limited capacity of visual attention (Verghese and Pelli 1992) the viewer is likely to miss some interesting content of the image, e.g. the flowers in the girl's hand. This is where EyeOfDetail comes into play, which invites the user to pay mindful attention to even unobtrusive elements by masking more salient details.

\section{ForgetMeNot (screensaver)}

ForgetMeNot is a screensaver that randomly chooses pictures from a selected folder and displays them with various degrees of blurring on the user's screen during idle time. The images are replaced after a couple of seconds. The aim of the application is to provide the observer with cues to encourage them to proactively remember an event. Cues are given by the degree of de-blurring of the image (in contrast to EyeOfDetail the whole picture is affected), and the degree of blurring is inversely related to time passed.

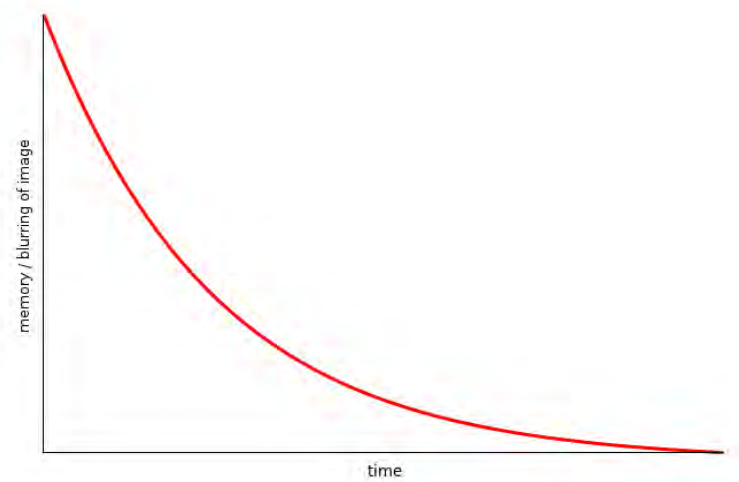

Figure 3: Forgetting and de-blurring curve. The images of ForgetMeNot get less blurry as our memory fades.
The main concept behind ForgetMeNot is that the degree of distortion is modelled as a function of time in the same fashion as Ebbinghaus' (1885) model for human memory retention. Figure 3 displays a typical forgetting curve as first described by Ebbinghaus. It visualizes how the content of human memory fades away over time.

In ForgetMeNot, the curve also indicates the degree of distortion of the images, the rationale being that when images are fresh in memory fewer cues are needed. Hence, a freshly shot picture will be shown in a very blurry fashion. The more time passes by the clearer the images get (see Figure 4). Hence as images in our mind fade away and become cloudy, ForgetMeNot will reveal more and more details as retrieval cues in the randomly displayed images (noticeable over weeks). The application will therefore help compensate for loss of memory by off-loading to the clearer photos. It also provides an element of serendipity and surprise in its random selection.
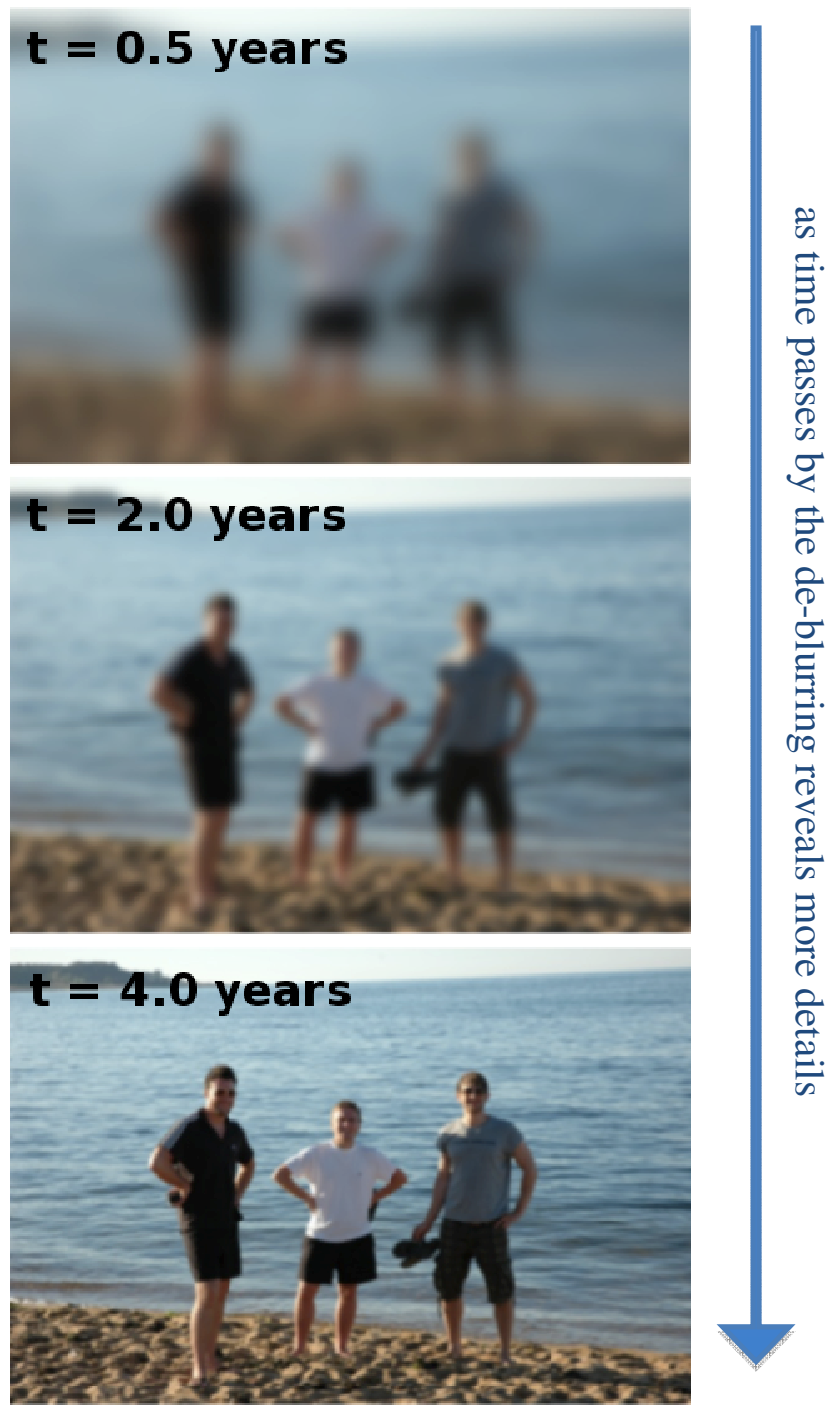

Figure 4: Three snapshots of ForgetMeNot. As days and weeks pass by the image will get less blurry. 
ForgetMeNot was primarily designed for stimulating the observer's memory. Due to the strong distortion of new pictures there is enough space for the mind to make up the story behind the photo. The application requires the observer to "participate in making meaning", because of its ambiguous display (Gaver et. al. 2003, p.235).

This strategy is also supported by a study incorporating images taken by the Microsoft SenseCam device. Here test participants reported that the distortion of the SenseCam images eventually turned out to be a benefit; because they were incomplete, participants had to do more work to fill in the details and this promoted reflection on the pictures (Fleck and Fitzpatrick 2009, 2010).

\section{BehindTheCamera (photo taking application)}

BehindTheCamera takes a different approach to adding information to photos. It takes advantage of the emerging trend for many smart-phones to include two cameras, one camera on the back of the phone and one additional front camera, principally designed for video-phone calling. BehindTheCamera is a mobile phone application that triggers front and rear camera at the same time when the user is taking a picture. As a consequence, the photo not only contains the target image, but the photographer as well (see Figure 5).

The initial motivation came from a recent experience by the first author who found an old photograph showing a group of young people who were obviously enjoying themselves in front of the Eiffel Tower. What the picture did not show was the person who took the image, of course. This led to serious pondering in this instance, because the photographer could not be recalled. BehindTheCamera was designed to prevent situations like the one described above.

Figuratively, BehindTheCamera conforms to Langer's definition of a mindful state of being, because it allows for more than one perspective and it is welcoming new information (1989, p.62). Fleck and Fitzpatrick (2010) also talk about the value of seeing from different perspectives to support reflection. Hence, we hypothesise that the application eventually will be able to promote a deeper experience for the observer in contrast to just viewing a regular image.

We also hypothesise that BehindTheCamera can provide valuable retrieval cues, which are of great importance for recollection and remembering (Tulving and Pearlstone 1966). The photographer not only possesses the image, but also a selfportrait of him/her taking the image. This additional portrait can potentially trigger different memories, because it provides the observer with additional retrieval cues. Recalling the way the photographer felt at that instant, for example, can support recalling the whole context. Emotional states not only have an effect on memory encoding, they are also able to facilitate memory retrieval (Buchanan 2007).

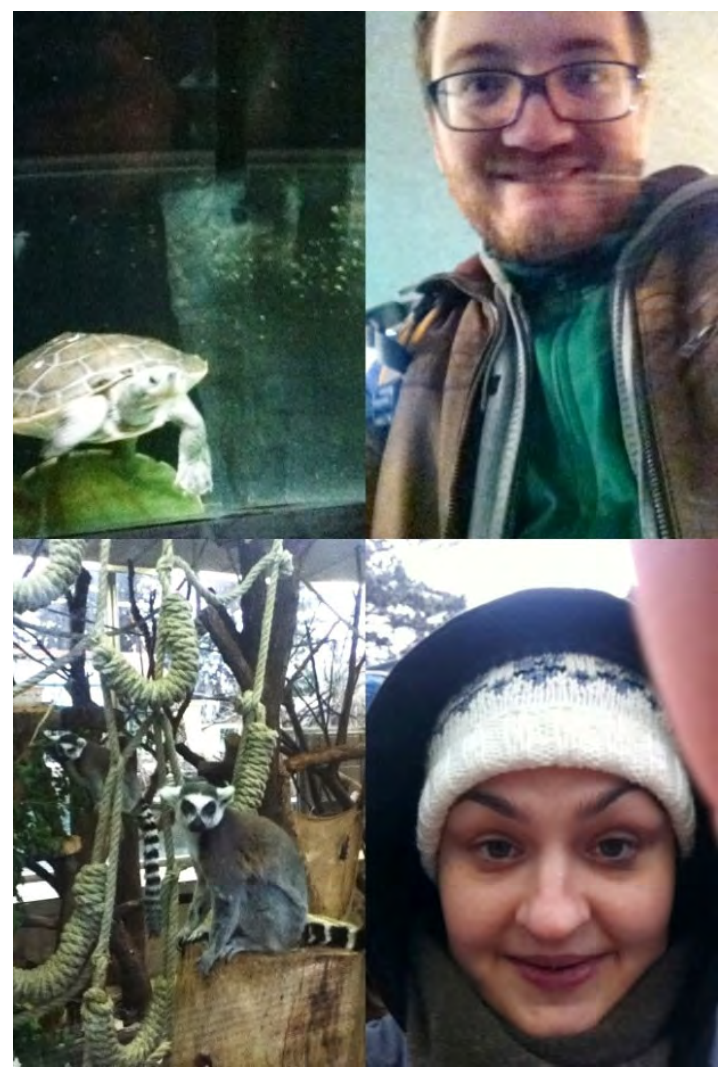

Figure 5: BehindTheCamera - photo taking application. Left side: motif. Right side: photographer.

The aim is that this simple application will deliver interesting meta-information and insights, especially as time passes by. BehindTheCamera draws a more complete picture of the whole scene. This is illustrated by the images of Figure 5, for example, which capture the interplay between animals and people.

\section{PILOT STUDIES}

To support our work in progress and gain initial feedback, we conducted small pilot studies with each of the three applications. We report on each of these in turn.

The first pilot study evaluated the EyeOfDetail application. We conducted a qualitative study using an image of our $\mathrm{HCl}$ group on a trip and recruiting eight test participants (TP), aged between 25 and 37 , who were all present in the photo. Hence all of the participants attended the trip, knew the image and thus had a personal relation to the picture. 
The participants were divided into two groups (2 females and 2 males each). Group A was asked to simply view the image displayed in Figure 6 (top). Group B had to use the EyeOfDetail application to explore the same image (Figure 6, middle). Each participant received the same instructions, regardless to which group the person belonged: Talk me through the image and any thoughts you have around it. You can take as much time as you like - we have up to 10 minutes. If you can think out loud for me that would be great.

The interviews were audio recorded. Additionally, the path along which the user moved his viewing window or clear spot with the EyeOfDetail version was tracked (see Figure 6, bottom). After participants finished viewing the picture the image viewers were switched. Hence, in the end every participant was exposed to both treatments (EyeOfDetail image viewer and a regular nonblurred image viewer).

In terms of results, most participants liked the EyeOfDetail application. However, one participant (TP8) thought it was dull and preferred the complete non-blurred image. Both groups spent considerably more time using the EyeOfDetail application as shown in Table 1.

\begin{tabular}{|l|l|l|}
\hline & Std viewer (mins) & EyeOfDetail (mins) \\
\hline Group A & $3: 23$ & $5: 12$ \\
\hline Group B & $2: 05$ & $4: 24$ \\
\hline A\&B & $2: 44$ & $4: 48$ \\
\hline
\end{tabular}

Table 1: Mean time spent viewing the images.

This is reassuring, because we actually wanted to slow the observer down. Moreover, we observed a tendency in group B to describe details whereas participants of group A more often talked about the image and trip in general. Exemplary statements of group A were "The image is not even" (TP5) and "Taking the trip was a good idea" (TP8). On the other hand, test participants using EyeOfDetail stated: "What is the name again of the guy from PhD school and where is Peter?" (TP1) and "I moved from person to person and thought about what is connecting me with each person." (TP6). Furthermore, TP7 reported on the way people held their hands. TP6 found herself talking about clothes, which according to her usually doesn't bother her at all. Also, two participants were attracted by the play between bright lights and shadow within the leaves and enjoyed exploring the treetops (see scan path of Figure 6, bottom) and experienced EyeOfDetail as "more intense" (TP1). This kind of openness (paying attention to the clothes, examining the treetops) nicely mirrors the "welcoming new information" criteria for mindfulness by Langer (1989, p. 62).
In another pilot study, ForgetMeNot was installed on the PC of one test participant for ten days. The number of images randomly displayed was restricted to five photographs involving her that were older than three years and provided by a friend. We adjusted the curve for de-blurring to reveal the content of the images within the ten days time-period. The modifications towards the original version were applied in order to get initial user feedback within a shorter range of time.

Here we highlight one interesting finding around the similarity of memories. The photo displayed the participant sitting at a table. The participant "could have sworn when looking at the blurred image" that she remembered clearly and was "one hundred percent accurate" in recounting that it was her favourite restaurant and recognizing roughly the

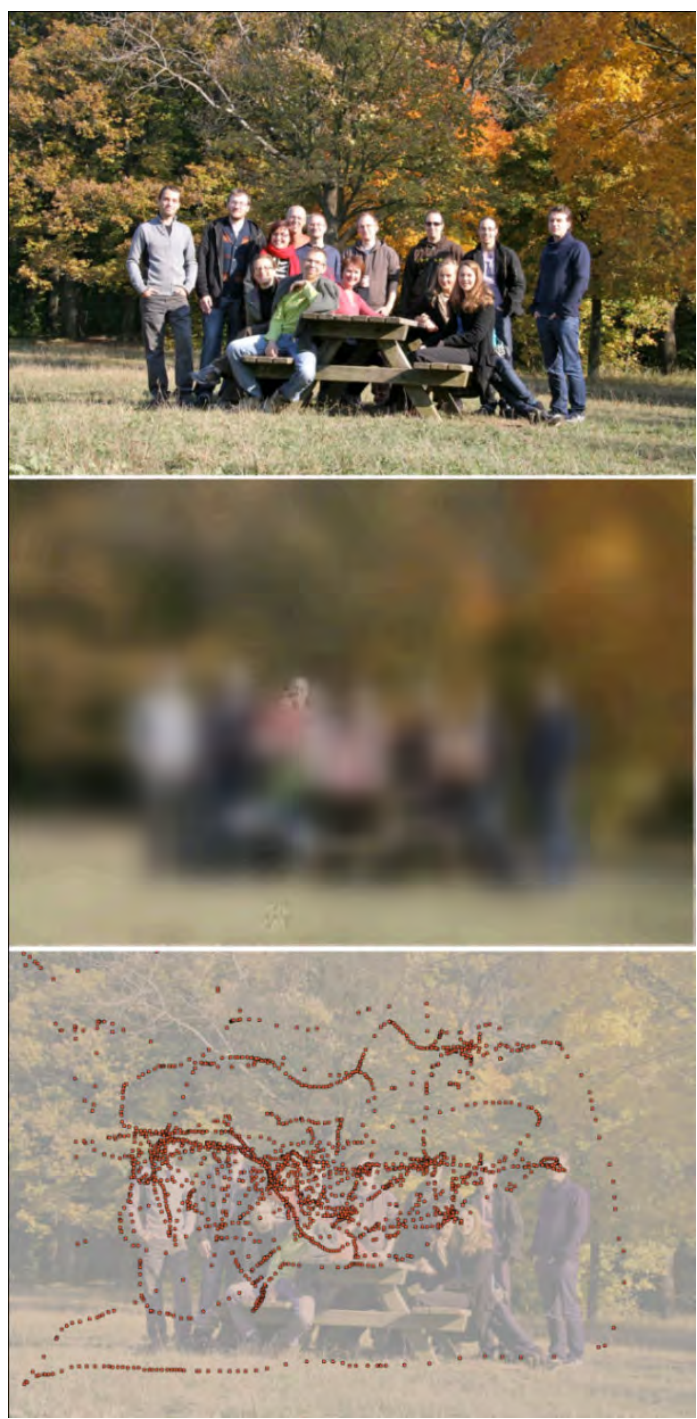

Figure 6: Test image for pilot study (top). Test image as displayed by EyeOfDetail (middle). Scan path of a test participant (bottom).

date of the image. She was later very surprised, as the image became less blurry, to realise that the 
photo was actually taken in her garden not in the restaurant.

It can be argued that the image opened up a rich space for remembering two events. It might also demonstrate an occurrence of a false memory, and a propensity to believe that memory is accurate. A vast amount of psychology literature deals with people's disposition to blindly believe in false memories (e.g. Loftus and Loftus 1980).

The third application, BehindTheCamera was tested by one of the authors and a friend. The application was used during a visit to the zoo. Two snapshots of this occasion are displayed in Figure 5. The author's friend commented that the animal pictures will surely lose interest as time passes by. On the other hand, the images of the photographers might gain interest in the future. Further analysis is still to be conducted.

\section{FUTURE WORK}

In this paper we proposed three ideas for augmenting digital photos for recollection, reminiscing and reflection. We demonstrated opportunities 'to get more out' of digital images with little technical effort. Also, we have pointed out theories from psychology that informed our implementations. While the small pilot tests demonstrated some potential value, further work is needed to involve more users in more extensive studies to explore the true potential of augmenting photos in these ways. We plan to setup additional user studies for getting feedback on the usefulness of the applications, to get further inspiration and to obtain a deeper insight on how people will incorporate our systems for recollecting, reminiscing and reflecting. Questions such as "how long should the screensaver display the images", "what is the best level of distortion" or "should some of the applications be implemented for tablet computers" might also be worth looking at. The aim is to understand the observer's needs for a technology that assists the process of remembering. We regard the concepts introduced in this paper as a general starting point for our exploration of how media content can be modified and augmented to support reminiscing, recollection and reflection.

\section{REFERENCES}

Banks R. and Sellen A. (2009) Shoebox: mixing storage and display of digital images in the home. In Proc of TEl2009, pp.35-40. ACM Press, NY. Brown, K.W. and Ryan, R.M. (2003) The benefits of being present: mindfulness and its role in psychological well-being. $J$ of Personality and Social Psychology, 84(4), pp.822-848.
Buchanan, T.W. (2007) Retrieval of emotional memories. Psychological Bulletin, 133(5), pp.761779.

Ebbinghaus, H. (1885; reprint 1964) Memory: $A$ Contribution to Experimental Psychology, Dover, NY.

Fleck, R. and Fitzpatrick, G. (2010) Reflecting on Reflection: Framing a Design Landscape. In Proc of OZCHI2010, CHISIG, pp. 216-223.

Fleck, R. and Fitzpatrick, G. (2009). Teachers' and tutors' social reflection around SenseCam images. Int $J$ of Human-Computer Studies, 67(12), pp.10241036.

Frohlich, D., Adams G. and Tallyn, E. (2000) Augmenting Photographs with Audio. Personal Technologies, 4(4), pp. 205-208.

Frohlich, D., Kuchinsky, A., Pering, C. Don, A. and Ariss, S. (2002) Requirements for photoware. In Proc of CSCW2002, pp.166-175. ACM Press NY. Gaver, W., Beaver, J. and Benford, S., (2003) Ambiguity as a resource for design. In Proc of CHI2003, pp.233-240. ACM Press, NY.

Havighurst, R.J. and Glasser, R., 1972. An exploratory study of reminiscence. Journal of Gerontology, 27(2), pp.245-253.

Kirk, D.S., Sellen, A.J., Rother, C. and Wood, K.R. (2006) Understanding Photowork. In Proc. Of CHI2006, pp.761-770. ACM Press, NY.

Langer, E. J., (1989) Mindfulness, Da Capo Press, Cambridge, MA.

Loftus, E.F. and Loftus, G.R., 1980. On the Permanence of Stored Information in the Human Brain. American Psychologist, 35(5), pp.409-420.

Peesapati, S.T., Schwanda, V., Schultz, J., Lepage, M., Jeong, S. and Cosley, D., (2010). Pensieve: supporting everyday reminiscence. In Proc of CHI2010, pp.2027-2036. ACM Press NY.

Sellen, A.J., Fogg, A., Aitken, M., Hodges, S. Rother, C. and Wood, K. (2007) Do life-logging technologies support memory for the past?: an experimental study using sensecam. In Proc of CHI2007. pp. 81-90. ACM Press, NY.

Sellen, A.J. and Whittaker, S. (2010) Beyond Total Capture: A Constructive Critique of Lifelogging. Communications of the ACM, 53(5), pp.70-77.

Tulving, E. and Pearlstone, Z. (1966) Availability versus accessibility of information in memory for words. J Of Verbal Learning And Verbal Behavior, 5(4), pp.381-391.

Uriu, D., Shiratori, N., Hashimoto, S., Ishibashi, S. And Okude N. (2009) CaraClock: An Interactive Photo Viewer Designed for Family Memories. In Proc CHI2009, pp.3205-3210. ACM Press, NY.

Verghese, P. and Pelli, D.G. (1992) The information capacity of visual attention. Vision Research, 32(5), pp.983-995.

Webster, J.D. and McCall, M.E. (1999) Reminiscence Functions Across Adulthood : A Replication and Extension. J of Adult Development, 6(1), pp.73-85. 